\title{
Heterogeneous catalysts for olefin polymerization: mathematical model for catalyst particle fragmentation
}

\author{
Adriano G. Fisch ${ }^{1}$, João H. Z. dos Santos ${ }^{2}$, Argimiro R. Secchi ${ }^{3}$, Nilo S. M. Cardozo ${ }^{4}$ \\ ${ }^{1}$ Chemical Engineering Department. Universidade Luterana do Brasil. \\ Av. Farroupilha 8001, 92425-900, Canoas, RS, Brazil. \\ ${ }^{2}$ Chemistry Institute. Universidade Federal do Rio Grande do Sul. \\ Av. Bento Gonçalves 9500. 91501-970 Porto Alegre, RS, Brazil. \\ ${ }^{3} \mathrm{PEQ} / \mathrm{COPPE}$, Universidade Federal do Rio de Janeiro. \\ Centro de Tecnologia G-116, Cx.P. 68502, 21941-972, Rio de Janeiro, RJ, Brazil. \\ ${ }^{4}$ Chemical Engineering Department, Universidade Federal do Rio Grande do Sul. \\ Rua Eng. Luis Englert s/n. 90040-040 Porto Alegre, RS, Brazil.
}

*Corresponding author. E-mail: adriano.fisch@ulbra.edu.br. Tel.: +55 5134774000. 


\section{Mass Balances for Olefin Polymerization}

The mass balance was evaluated for sites (active and inactive) and polymer chain (live and dead) distributed over the catalyst particle. The kinetic mechanism is described in Table 1 of the article. Equation 20 of the article describes the mass balance employed. As neither sites nor polymer chains could move inside the particle, the respective terms that are related to the transport (diffusion and advection) of these compounds are null.

Population balance using the method of moments of the distribution was employed to solve the polymer chain distribution. Equation S1 presents the definition of moments and Equation S2 is the zero order moment, which is needed to solve the balance. Moments of higher order could be evaluated if the molecular mass averages are needed.

$$
\begin{aligned}
& \mu_{k}=\sum_{r=1}^{\infty}\left[r^{k} P_{r}\right] \\
& \mu_{0}=\sum_{r=1}^{\infty}\left[r^{0} P_{r}\right]=\sum_{r=1}^{\infty}\left[P_{r}\right]
\end{aligned}
$$

Equations S3-15 present the detailed treatment for getting the mass balances for sites, polymer chains, and reaction rates of reactants.

\section{$\underline{\text { Live (growing) polymer chain }}$}

$$
\begin{aligned}
& \frac{d\left[P_{1}\right]}{d t}=k_{p}[M][S]-k_{p}[M]\left[P_{1}\right]-k_{t \beta}\left[P_{1}\right]-k_{t H}[H]\left[P_{1}\right]-k_{d}[I]\left[P_{1}\right] \\
& \frac{d \sum_{r=2}^{\infty}\left[P_{r}\right]}{d t}=k_{p}[M] \sum_{r=2}^{\infty}\left[P_{r-1}\right]-k_{p}[M] \sum_{r=2}^{\infty}\left[P_{r}\right]-k_{t \beta} \sum_{r=2}^{\infty}\left[P_{r}\right]-k_{t H}[H] \sum_{r=2}^{\infty}\left[P_{r}\right]-k_{d}[I] \sum_{r=2}^{\infty}\left[P_{r}\right] \\
& \frac{d \sum_{r=1}^{\infty}\left[P_{r}\right]}{d t}=\frac{d\left[P_{1}\right]}{d t}+\frac{d \sum_{r=2}^{\infty}\left[P_{r}\right]}{d t}
\end{aligned}
$$




$$
\begin{aligned}
& \frac{d\left[\mu_{0}\right]}{d t}=\frac{d\left[P_{1}\right]}{d t}+\frac{d \sum_{r=2}^{\infty}\left[P_{r}\right]}{d t}= \\
& \quad=k_{p}[M][S]-k_{p}[M]\left[P_{1}\right]-k_{t \beta}\left[P_{1}\right]-k_{t H}[H]\left[P_{1}\right]-k_{d}[I]\left[P_{1}\right]+ \\
& \quad+k_{p}[M] \sum_{r=2}^{\infty}\left[P_{r-1}\right]-k_{p}[M] \sum_{r=2}^{\infty}\left[P_{r}\right]-k_{t \beta} \sum_{r=2}^{\infty}\left[P_{r}\right]-k_{t H}[H] \sum_{r=2}^{\infty}\left[P_{r}\right]-k_{d}[I] \sum_{r=2}^{\infty}\left[P_{r}\right]
\end{aligned}
$$

Simplifications

$$
\begin{aligned}
& k_{p}[M] \sum_{r=2}^{\infty}\left[P_{r-1}\right]-k_{p}[M]\left[P_{1}\right]-k_{p}[M] \sum_{r=2}^{\infty}\left[P_{r}\right]=0 \\
& k_{t \beta}\left[P_{1}\right]+k_{t \beta} \sum_{r=2}^{\infty}\left[P_{r}\right]=k_{t \beta} \sum_{r=1}^{\infty}\left[P_{r}\right]=k_{t \beta}\left[\mu_{0}\right] \\
& k_{t H}[H]\left[P_{1}\right]+k_{t H}[H] \sum_{r=2}^{\infty}\left[P_{r}\right]=k_{t H}[H] \sum_{r=1}^{\infty}\left[P_{r}\right]=k_{t H}[H]\left[\mu_{0}\right] \\
& k_{d}[I]\left[P_{1}\right]+k_{d}[I] \sum_{r=2}^{\infty}\left[P_{r}\right]=k_{d}[I] \sum_{r=1}^{\infty}\left[P_{r}\right]=k_{d}[I]\left[\mu_{0}\right]
\end{aligned}
$$

Replacing equations

$$
\frac{d\left[\mu_{0}\right]}{d t}=k_{p}[M][S]-k_{t \beta}\left[\mu_{0}\right]-k_{t H}[H]\left[\mu_{0}\right]-k_{d}[I]\left[\mu_{0}\right]
$$

\section{Active site S}

$$
\begin{aligned}
& \frac{d[S]}{d t}=k_{t \beta} \sum_{r=1}^{\infty}\left[P_{r}\right]+k_{t H}[H] \sum_{r=1}^{\infty}\left[P_{r}\right]-k_{p}[S][M]-k_{d}[S][I] \\
& \frac{d[S]}{d t}=k_{t \beta}\left[\mu_{0}\right]+k_{t H}[H]\left[\mu_{0}\right]-k_{p}[S][M]-k_{d}[S][I]
\end{aligned}
$$

\section{$\underline{\text { Inactive site }}$}

$$
\frac{d\left[S_{d}\right]}{d t}=k_{d}[S][I]+k_{d}[I] \sum_{r=1}^{\infty}\left[P_{r}\right]
$$


$\frac{d\left[S_{d}\right]}{d t}=k_{d}[S][I]+k_{d}[I]\left[\mu_{0}\right]$

\section{$\underline{\text { Reaction rates }}$}

$$
\begin{aligned}
& R_{M}=k_{p}[M \rrbracket S]-k_{p}[M] \sum_{r=1}^{\infty}\left[P_{r}\right]=k_{p}\left[M \rrbracket[S]-k_{p}\left[M \rrbracket \mu_{0}\right]\right. \\
& R_{H}=k_{t H}\left[H \rrbracket \mu_{0}\right] \\
& R_{I}=k_{d}[S \rrbracket I]+k_{d}\left[I \rrbracket\left[\mu_{0}\right]\right.
\end{aligned}
$$

The definitions of Equations S16-20 were employed getting the final set of dimensionless equations, which was implemented in the simulator.

$z=\frac{r}{R}$, dimensionless radial position

$B=\frac{K}{P_{\text {ref }}}$, dimensionless bulk modulus

$W=\frac{P}{P_{r e f}}$, dimensionless pressure

$G=\frac{m_{p o l}}{m_{r e f}}$, dimensionless polymer mass

$[X]_{a}=\frac{[X]}{[X]_{b u l k}}$, dimensionless reactant concentration 